\title{
Meta Analysis on Pregnancy and Perinatal Outcomes of Pregnant Women with COVID-19
}

\author{
Saraswati Basnet*
}

\begin{abstract}
Covid-19' was originated from the Wuhan, China, in December 2019, and it was also named as 2019-nCoV SARS-CoV-2. The corona virus 2 (SARS-CoV-2) is a newly discovered ribonucleic acid virus which is identified from the patients with unexplained pneumonia and severe in acute respiratory syndrome. This Meta-analysis aims to evaluate the effect on pregnancy, perinatal, and neonatal outcomes of pregnant women with Covid-19. This paper is mainly based on secondary data sources. Data has been collected from the reviews of international and national publications, such as journals, reports, articles, and e-resources published in April to November 2020. A formal extraction protocol workflow was used to measure Preferred Reporting Items for Systematic Review and Meta-Analysis Protocol (PRISMA-P). The findings show that 2099 newborns (including twins) were borned by 2093 pregnant women since the period of Covid-19. The result indicates that more than 74 percent of the pregnant women had given birth by cesarean section due to maternal Covid-19 complications and fetal distress. Most of the pregnant women have the following symptoms of Covid19, such as fever, cough, dyspnea, pneumonia, respiratory distress syndrome, premature delivery, etc; and neonates often have the following symptoms: respiratory distress pneumonia, bacterial pneumonia, neonatal death, and Covid-19 positive. This paper concludes that the pregnancy and perinatal outcome of pregnant women and neonates were varied, and their manifestation can range normal, mild, and severe.
\end{abstract}

Key Words: human gestation, neonatal asphyxia, respiratory syndrome, reproductive health, perinatal resection.

\section{Introduction}

This paper attempts to explore the meta-analysis on pregnancy and perinatal outcomes of pregnant women with COVID-19. Novel Corona virus disease -19 is an emerging disease with a rapid increase in cases and deaths since its first identification in

${ }^{*}$ Ms. Basnet is the Lecturer in Nursing currently working at Biratnagar Nursing Campus, IoM, Tribhuvan University, Nepal. 
Wuhan, China, in December 2019.Corona virus disease 2019 during pregnancy related information or illnesses associated with other highly pathogenic corona viruses (i.e., severe acute respiratory syndrome and the Middle East respiratory syndrome) might provide insights into corona virus disease 2019's effects during pregnancy. Corona viruses cause illness ranging in severity from the common cold to severe respiratory illness and death (Rasmussen, Smulian, Lednicky, Wen \& Jamieson, 2020).

World Health Organization (WHO) (2020) argues that people who are non-white, older, and overweight and have a pre-existing medical condition are more vulnerable to severe disease due to Covid-19 and also more likely to suffer severe health complications due to Covid-19. Pregnant women with Covid-19 were more likely to give birth prematurely and 1 in 4 of all babies born to women with Covid-19 was admitted to a neonatal unit, stillbirth and newborn death rates were low.

Clinical retrospectively review for nine pregnant women with Covid-19 pneumonia result reveals that the maternal symptoms of cough, myalgia, sore throat and malaise, lymphopenia $\left(<1.0 \times 10^{9}\right.$ cell/liter $)$ and fetal distress but the new born infants were tested negative for Covid-19 and there is no evidence of Covid -19 vertical transmission of mother to fetus (Chen, et al., 2020).

Nearly one-third of pregnant women with Covid-19 were hospitalized compared with just six percent of nonpregnant women. Pregnant women with Covid-19 were more likely to be admitted to the ICU and required mechanical ventilation compared with nonpregnant women. Among pregnancy $1.5 \%$ of pregnant women were admitted to the ICU compared with $0.9 \%$ of nonpregnant women; and $0.5 \%$ of pregnant women required mechanical ventilation compared with $0.3 \%$ of nonpregnant women (Ablow, 2020).

Yu et al., (2020) reported that clinical manifestations of pregnant women with Covid-19 were fever 86\%), cough (14\%), shortness of breath (14\%), and diarrhea $(14 \%)$. All patients had caesarean section within 3 days of clinical presentation with an average gestational age of 39 weeks plus 2 days. The overall outcomes of the pregnant women and neonates were good but one neonate was infected with SARS-CoV-2, 36 hours after birth.

Case-control study conducted by Li, et al., (2020) among 16 pregnant women with Covid-19 (Cases) and 18 pregnant women with suspected-Covid-19 (Controls) result shows that two pregnant women had vaginal delivery and rest were cesarean. Few pregnant women had respiratory problems as fever and cough but most of pregnant women had Covid-19 pneumonia none experienced respiratory failure during hospitalization. Eighteenth (18\%) percent of pregnant women with confirmed Covid-19 had pneumonia and $16 \%$ pregnant women with suspected Covid-19 had preterm delivery due to maternal complications which were significantly higher than in the 
control group. In terms of the newborns, absence of Covid-19 infection and severe neonatal complications.

In United Kingdom, study conducted by Antoun, Taweel, Ahmed, Patni \& Honest (2020) agreed that 22 pregnant women with Covid-19, the pregnancy and perinatal outcome was $65.2 \%$ of the pregnant women presented mild, $(8.7 \%)$ of pregnant women presented moderate and $34.8 \%$ of pregnant womenpresented severe symptoms. Among them $17.4 \%$ (out of 19 pregnant women) developed severe adult respiratory distress syndrome complications requiring ICU support (4.3\%), (36.8\%) had preterm birth, $15.8 \%$ developed adult respiratory distress syndrome before delivery, $10.5 \%$ had pre-eclampsia and $84 \%$ of patients delivered by C-section. Out of the 20 newborns, 18 were singletons with a set of twin. Among them neonate had bacterial pneumonia (one), Asphyxia (one) and were admitted to hospital due to infection and others were normal.

In India, Hassan, Muzamil \& Banday (2020) claims that among 38 pregnant women with Covid-19 pregnancy and maternal perinatal (the period commences at 22 completed weeks) outcomes were $79 \%$ term delivery, $21 \%$ preterm delivery. The mode of delivery of pregnant women with Covid-19 were (60\%) done cesarean section, $(39.5 \%)$ were vaginal delivery, 2 pregnant women were risk of diabetes and 1 were risk of pregnancy induced hypertension, 1 mother was ICU admission and 1 died. In term of neonatal outcome were $26.3 \%$ of newborns had meconium (stool of fetus) stained, $29 \%$ newborns had fetal distress, 29\% neonatal had ICU admissions, $13.5 \%$ had neonatal deaths, $(2.7 \%)$ had intra uterine death, 2.63\% had Vertical transmission, 2.6\% had lab characteristics anemia, 21\% had thrombocytopenia, 21\% had SARS CoV-2 positive (by RT-PCR), $100 \%$ were presented with heart failure and shock at 17th day of life.

Similarly, in Nepal comparative observational study was conducted by K.C., et al., (2020) shows that the proportion of who had a complication during admission increased from $6.7 \%$ to $8.7 \%$ before and during lockdown $(\mathrm{p}=0.0126)$. The proportion of women whose labor was induced increased by $17.1 \%$ to $32.1 \%$ before and during the lockdown $(\mathrm{p}=0.0001)$. The proportion of women who had caesarean section increased by $24.5 \%$ to $26.2 \%$ before and during lockdown ( $\mathrm{p}=0.0075)$. The proportion of babies borned preterm (before 37 weeks) increased from $16.7 \%$ to $20.0 \%$ before and during lockdown $(\mathrm{p}=0 \cdot 0016)$. The institutional stillbirth (the death or loss of a baby before or during delivery) rate was increased by 14 to 21 per 1000 live births. The neonatal (birth to 28 days' baby) mortality rate was increased by 13 deaths to 40 deaths per live births before and during lockdown.

\section{Objective}


The objective of this Meta analysis was to evaluate the pregnancy and perinatal outcome of pregnant women with Covid-19.

\section{Materials and Methods}

Design: Meta analysis was done by using published national and international journals, articles and reports.

Inclusion criteria: The inclusion criteria of study were developed with carefully examined published national and international research articles, reports which were searched from the different databases such as Google, Google scholar, Pub med, and Hinari from April 2020 to November, 2020. The main focus was done on Pregnancy, perinatal and neonatal outcomes of pregnant women with Covid-19. The review was done only on; a) English language b) involved samples of at least 7 to 675 subjects c) eligible diverse research design

Exclusion criteria: The articles related to unreliable data, incomplete information, experimental studies and randomized trial were excluded from data.

Search Strategy: The research articles related on pregnancy, perinatal and neonatal outcomes of pregnant women with Covid-19 were searched for data purpose. During acquisition of data, key words were used as pregnancy, perinatal and neonatal outcome of pregnant women with Covid-19. Hand-searched (manual search) such as the reference lists of all identified studies and key journals in the related field was developed. About more than 193 reports were reviewed thoroughly and captured the required theme. The most common studies were pregnancy, perinatal and neonatal outcome of pregnant women with Covid-19: The preliminary analysis and searched article's study period was ranging from 2 weeks to 3 months and less than 1 year. Data Extraction, analysis and synthesis: While searching, the main key words were used as pregnancy, perinatal and neonatal outcomes of pregnant women with Covid-19. The total searched articles were 193 which were published from December 2019 to November 2020. The search engines were Google, scholarly Google Pub med and Hinari through the internet database. A formal extraction protocol was developed as the PRISMA-P (Preferred Reporting Items for Systematic Review and Meta analysis Protocol) workflow which was used by Moher D, et al. 2015 that were checked and revised two or three times. Among 193 articles, 181 articles were excluded from the protocol for the following reason i.e. not relevant, unavailability of total result and incomplete result. Eligibility and inclusion criteria or content related topic of this analysis weren't provided. Only 12 full text articles related to Pregnancy, perinatal and neonatal outcome of pregnant women with Covid-19 were kept. The total sample size of 12 articles was 2093. 


\section{Results}

Result analysis of Meta analysis according to PRISMA-P (Preferred Reporting Items for Systematic Review and Meta analysis Protocol) as below.

\begin{tabular}{|c|c|c|c|}
\hline $\begin{array}{l}\text { Record identified } \\
\text { through database } \\
\text { searching }\end{array}$ & & \\
\hline \multirow{3}{*}{$\begin{array}{l}\text { Reaming record screened } \\
\text { after } 15828 \text { duplicates } \\
\text { removed } \downarrow \\
(\mathrm{n}=2193)\end{array}$} & \multirow{2}{*}{$\begin{array}{l}\text { Record excluded } \\
(\mathrm{n}=2000)\end{array}$} & & \\
\hline & & & \\
\hline & \multirow{2}{*}{\multicolumn{3}{|c|}{$\begin{array}{l}\text { Full text article excluded }(\mathrm{n}=181) \\
\text { Reason: } \text { not relevant }=80 \\
\text { Total result not available }=88 \\
\text { Not accessible }=9 \\
\text { Not available complete }\end{array}$}} \\
\hline $\begin{array}{l}\text { Full text article } \\
\text { assessed } \$ \text { or eligibility } \\
(n=193)\end{array}$ & & & \\
\hline $\begin{array}{l}\text { Studies included in } \\
\text { narrative synthesis } \\
(n=12)\end{array}$ & & & \\
\hline
\end{tabular}

Results of the reviewed Analysis: Overall 12 articles were reviewed and analysis done. Meta analysis was done among 2093 pregnant women with Covid-19 and 2099 born neonates' (including twins) pregnancy, perinatal maternal characteristics and neonatal outcomes complications. More than two third (74.16\% or 1549) of pregnant women with Covid-19 reported cesarean section, less than one third (21.81\% or 844$)$ of pregnant women reported vaginal delivery and only least (4.02\% or 12) pregnant women with Covid-19 reported spontaneous fetus loss in $1^{\text {st }}, 2^{\text {nd }}$ and $3^{\text {rd }}$ trimester. The most common symptoms noticed on pregnant women with Covid-19 were fever, cough, dyspnea, pneumonia, severe respiratory distress syndrome, premature delivery, eclampsia, fetal distress, preterm birth, severe respiratory distress which leaded to ICU admission and ventilator support. The common symptoms of neonates were respiratory distress pneumonia, bacterial pneumonia, low birth, rash asphyxia, DIC, perinatal death and Covid-19 positive. Most of studies were shows that there was no vertical and horizontal study.

\section{Table 1}

Meta analysis according to Author, Country, Sample size, design of perinatal outcomes

\begin{tabular}{|l|l|l|l|}
\hline Author & $\begin{array}{l}\text { Countr } \\
\mathbf{y}\end{array}$ & $\begin{array}{l}\text { study design/ } \\
\text { Sample size }\end{array}$ & Pregnant women's Prenatal outcome \\
\hline $\begin{array}{l}\text { (Liao, He, } \\
\text { Gong, Yang, } \\
\text { Zhou, \& Li, }\end{array}$ & China & $\begin{array}{l}\text { Comparative } \\
\text { study /10 } \\
\text { pregnant with }\end{array}$ & $\begin{array}{l}\text { No significance difference in age, postpartum } \\
\text { hemorrhage, perinatal resection between } 2 \\
\text { groups. }\end{array}$ \\
\hline
\end{tabular}




\begin{tabular}{|c|c|c|c|}
\hline 2020). & & Covid-19 & \\
\hline (Liu, 2020), & china & $\begin{array}{l}\text { retrospectively } \\
\text { reviewed/ } 15 \\
\text { Pregnant } \\
\text { women with } \\
\text { Covid-19 }\end{array}$ & $\begin{array}{l}\text { Absence of aggregative symptoms, CT features } \\
\text { of Covid-19 pneumonia and better recovery. }\end{array}$ \\
\hline $\begin{array}{l}\text { (Favre, } \\
\text { Pomar, } \\
\text { Musso \& } \\
\text { Baud, 2020), }\end{array}$ & China & $\begin{array}{l}\text { Retrospective } \\
\text { study/ } \\
29 \text { pregnant } \\
\text { with Covid-19 } \\
\text { positive }\end{array}$ & $\begin{array}{l}14 \text { pregnant women experienced mild symptoms } \\
\text { and } 15 \text { were free from symptoms of Covid-19. } \\
\text { Eight had fever, } 9 \text { were coughing, } 3 \text { had } \\
\text { shortness of breath, } 2 \text { had diarrhea, } 1 \text { had } \\
\text { pregnancy related complication, } 27 \text { had done } \\
\text { cesarean section, }\end{array}$ \\
\hline $\begin{array}{l}\text { (Blitz, et al., } \\
\text { 2020) }\end{array}$ & $\begin{array}{l}\text { New } \\
\text { York, } \\
\text { Americ } \\
\text { a }\end{array}$ & $\begin{array}{l}\text { Case series } \\
\text { evaluation/ } \\
462 \text { pregnant } \\
\text { With Covid- } \\
19 \text {, but case } \\
\text { seen on } 70 \\
\text { pregnant } \\
\text { women with } \\
\text { severe Covid- } \\
19 .\end{array}$ & $\begin{array}{l}70(15 \%) \text { had severe COVID19, out of these } 70 \\
\text { patients, a total of } 13(19 \%) \text { were admitted to } \\
\text { the ICU, }(15 \%) \text { died, and } 11(85 \%) \text { were } \\
\text { discharged. } 7 \text { women }(54 \%) \text { who delivered, } 5 \\
(71 \%) \text { were urgent cesarean deliveries due to } \\
\text { respiratory decomposition, } 1 \text { was an emergent } \\
\text { cesarean delivery for cord prolapsed, 1delivered } \\
\text { vaginally \& } 4(57 \%) \text { preterm births. }\end{array}$ \\
\hline $\begin{array}{l}\text { (Prabhu, et } \\
\text { al., 2020) }\end{array}$ & $\begin{array}{l}\text { New } \\
\text { York, } \\
\text { Americ } \\
\text { a }\end{array}$ & $\begin{array}{l}\text { perspective } \\
\text { cohort study/ } \\
675 \text { pregnant } \\
\text { women with } \\
\text { Covid-19 } \\
\& \text { without } \\
\text { Covid-19 }\end{array}$ & $\begin{array}{l}\text { 10.4\% were SAR CoV2 infection } \& 78.6 \% \text { were } \\
\text { symptomatic. Cesarean section: symptomatic } \\
46.7 \% \text {, asymptomatic } 45.5 \% \text { \& without Covid- } \\
1930.5 \% \text {. There wasn't any ICU admission and } \\
\text { maternal death due to Covid- } 19 \text {. }\end{array}$ \\
\hline $\begin{array}{l}\text { (Antoun, } \\
\text { Taweel, } \\
\text { Ahmed, } \\
\text { Patni \& } \\
\text { Honest } \\
\text { 2020) }\end{array}$ & $\begin{array}{l}\text { United } \\
\text { Kingdo } \\
\mathrm{m} \\
(\mathrm{UK})\end{array}$ & $\begin{array}{l}22 \text { pregnant } \\
\text { women with } \\
\text { Covid-19 }\end{array}$ & $\begin{array}{l}\text { Presented Covid-19 symptoms: } 65.2 \% \text { mild, } \\
8.7 \% \text { moderate } 34.8 \% \text { severe. } 19.4 \% \text { severe } \\
\text { respiratory distress so } 4.3 \% \text { required ICU } \\
\text { support, } 36.8 \% \text { had preterm both and } 84 \% \\
\text { delivered cesarean section. }\end{array}$ \\
\hline $\begin{array}{l}\text { (Knight, et } \\
\text { al., 2020) }\end{array}$ & $\begin{array}{l}\text { United } \\
\text { Kingdo } \\
\mathrm{m}\end{array}$ & $\begin{array}{l}\text { Cohort study/ } \\
427 \text { pregnant } \\
\text { women with }\end{array}$ & $\begin{array}{l}266(66 \%) \text { women gave birth or had pregnancy } \\
\text { loss, } 196(73 \%) \text { gave birth at a term. forty one } \\
(10 \%) \text { women had admitted to hospital for }\end{array}$ \\
\hline
\end{tabular}




\begin{tabular}{|c|c|c|c|}
\hline & (UK) & $\begin{array}{l}\text { Covid-19, (3 } \\
\text { group*) }\end{array}$ & respiratory support, five (1\%) women died. \\
\hline $\begin{array}{l}\text { (Marin } \\
\text { Gabriel, et } \\
\text { al., 2020), }\end{array}$ & Span & $\begin{array}{l}\text { descriptive } \\
\text { study/ } \\
242 \text { pregnant } \\
\text { women with } \\
\text { Covid-19 }\end{array}$ & $\begin{array}{l}26 \% \text { of women delivered by cesarean section, } \\
33 \% \text { women had cough, } 29.2 \% \text { had fever, } \\
46.6 \% \text { of women delivered by prematurity. }\end{array}$ \\
\hline $\begin{array}{l}\text { (Zimmerman } \\
\text { n\& Curtis, } \\
\text { 2020). }\end{array}$ & China & Case series & $\begin{array}{l}\text { Cesarean section was } 88 \% \text {, fetal distress was } \\
31 \% \text {, and preterm delivery was } 38 \% \text {, premature } \\
\text { rupture of membrane was } 12 \% \text {, pre-eclampsia } \\
\text { was } 3 \% \text {, abnormal umbilical cord was } 3 \% \text { and } \\
\text { ICU admission and mechanical ventilator was } \\
3 \% \text {. }\end{array}$ \\
\hline $\begin{array}{l}\text { (Zaigham \& } \\
\text { Andersson, } \\
\text { 2020), }\end{array}$ & Sweden & $\begin{array}{l}\text { Systematic } \\
\text { reviewed } \\
108 \text { pregnant } \\
\text { with Covid-19 }\end{array}$ & $\begin{array}{l}\text { Maternal complication on } 3^{\text {rd }} \text { trimester such as } \\
\text { fever was } 68 \%, 91 \% \text { of women had cesarean, } 3 \\
\text { mothers were admitted in intensive care unit } \\
\text { (ICU). }\end{array}$ \\
\hline $\begin{array}{l}\text { (Hassan, } \\
\text { Muzamil \& } \\
\text { Banday } \\
\text { 2020) }\end{array}$ & India, & $\begin{array}{l}\text { Observational } \\
\text { study/38 } \\
\text { pregnant } \\
\text { women with } \\
\text { Covid-19 }\end{array}$ & $\begin{array}{l}79 \% \text { was term delivery } \& 21 \% \text { was preterm } \\
\text { delivery. }(60 \%) \text { done cesarean section, }(39.5 \%) \\
\text { were vaginal delivery, } 1 \text { mother was admitted in } \\
\text { ICU and } 1 \text { died.. }\end{array}$ \\
\hline $\begin{array}{l}\text { (KC, et al., } \\
2020) \text {, }\end{array}$ & Nepal & $\begin{array}{l}\text { Perspective } \\
\text { observational } \\
\text { study } \\
21760 \\
\text { pregnant } \\
\text { mothers } \\
\text { without Covid- } \\
19\end{array}$ & $\begin{array}{l}\text { Weekly birth was decreased } 52.4 \% \text { in Covid-19. } \\
\text { Vaginal births } 10453 \text { was observed over the } \\
\text { study period with } 8228(78 \cdot 7 \%) \text { before } \\
\text { lockdown and } 2225(21 \cdot 3 \%) \text { during lockdown. } \\
\text { Caesarean section increased from } 24 \cdot 5 \% \\
(\mathrm{n}=3234) \text { before lockdown to } 26 \cdot 2 \%(\mathrm{n}=1879) \\
\text { during lockdown ( } \mathrm{p}=0.0075) \text {. }\end{array}$ \\
\hline
\end{tabular}

*Total 11article's study populations were 2093 pregnant women with Covid-19 but Nepal study population was not included. 


\section{Table 2}

Meta analysis according to Author, Country, Sample size, Design of Neonatal outcomes*

\begin{tabular}{|c|c|c|c|}
\hline Author & $\begin{array}{l}\text { Countr } \\
\mathbf{y}\end{array}$ & $\begin{array}{l}\text { Design \& Sample } \\
\text { size }\end{array}$ & Neonatal outcome \\
\hline $\begin{array}{l}\text { (Liao, He, } \\
\text { Gong, } \\
\text { Yang, Zhou, } \\
\& \text { Li, 2020). }\end{array}$ & China & $\begin{array}{l}\text { Comparative study } \\
/ 10 \text { pregnant with } \\
\text { Covid-19 }\end{array}$ & All of neonate was Covid-19 negative. \\
\hline (Liu, 2020), & China & $\begin{array}{l}\text { retrospectively } \\
\text { reviewed/ }\end{array}$ & $\begin{array}{l}\text { Covid-19 infection wasn't present in } \\
\text { newborn babies. }\end{array}$ \\
\hline $\begin{array}{l}\text { (Favre, } \\
\text { Pomar, } \\
\text { Musso \& } \\
\text { Baud, } \\
\text { 2020), }\end{array}$ & China & $\begin{array}{l}\text { Retrospective study/ } \\
29 \text { pregnant with } \\
\text { Covid-19 positive }\end{array}$ & $\begin{array}{l}\text { Among } 30 \text { neonates, } 18 \text { were hospitalized } \\
\text { for quarantine and care, } 12 \text { neonates were } \\
\text { discharged with normal condition, } 5 \\
\text { neonates were admitted due to covid-19, } 12 \\
\text { were hospitalized with pneumonia with } \\
\text { cough and } 2 \text { neonates had SARs-Cov2 } \\
\text { specific. }\end{array}$ \\
\hline $\begin{array}{l}\text { (Zimmerma } \\
\text { nn\& Curtis, } \\
\text { 2020). }\end{array}$ & China & Case series & $\begin{array}{l}\text { Neonatal pneumonia (18\%), disseminated } \\
\text { intravascular coagulation (3\%), asphyxia } \\
(2 \%) \text { and } 2 \text { perinatal deaths. Four neonates } \\
\text { ( } 3 \text { with pneumonia) have been reported to } \\
\text { be SARS-CoV-2 positive. }\end{array}$ \\
\hline $\begin{array}{l}\text { (Blitz, et al., } \\
2020)\end{array}$ & $\begin{array}{l}\text { New } \\
\text { York, } \\
\text { Americ } \\
\text { a }\end{array}$ & $\begin{array}{l}\text { Case series } \\
\text { evaluation/ } \\
462 \text { pregnant With } \\
\text { Covid-19, but case } \\
\text { seen on } 70 \text { pregnant } \\
\text { women with severe } \\
\text { Covid-19. }\end{array}$ & $(57 \%)$ preterm births. \\
\hline $\begin{array}{l}\text { (Prabhu, et } \\
\text { al., 2020) }\end{array}$ & $\begin{array}{l}\text { New } \\
\text { York, } \\
\text { Americ } \\
\text { a }\end{array}$ & $\begin{array}{l}\text { perspective cohort } \\
\text { study/ } 675 \text { pregnant } \\
\text { women with Covid- } \\
19 \& \text { without Covid- } \\
19\end{array}$ & $\begin{array}{l}\text { Absence of Covid-19 positive \& placental } \\
\text { pathology: fetal vascular malperfusion, } \\
\text { thrombi in fetal vessels. }\end{array}$ \\
\hline
\end{tabular}




\begin{tabular}{|c|c|c|c|}
\hline $\begin{array}{l}\text { (Antoun, } \\
\text { Taweel, } \\
\text { Ahmed, } \\
\text { Patni \& } \\
\text { Honest } \\
\text { 2020) }\end{array}$ & $\begin{array}{l}\text { United } \\
\text { Kingdo } \\
\mathrm{m} \\
\text { (UK) }\end{array}$ & $\begin{array}{l}22 \text { pregnant women } \\
\text { with Covid-19/ Case } \\
\text { Series }\end{array}$ & $\begin{array}{l}\text { There was bacterial pneumonia in } 1 \text { baby, } \\
\text { resuscitation \& intubation was done in } 1 \\
\text { baby and admitted special baby care Unit, } \\
37 \% \text { of severe Covid-19 mother's baby } \\
\text { hadn't Covid - } 19 \text { positive. There is no } \\
\text { evidence of Covid-19 vertical transmission } \\
\text { of mother to baby. }\end{array}$ \\
\hline $\begin{array}{l}\text { (Knight, et } \\
\text { al., 2020) }\end{array}$ & $\begin{array}{l}\text { United } \\
\text { Kingdo } \\
\mathrm{m} \\
\text { (UK) }\end{array}$ & $\begin{array}{l}\text { Cohort study/ } \\
427 \text { pregnant women } \\
\text { with COvid-19 }\end{array}$ & $\begin{array}{l}\text { Twelve }(5 \%) \text { of } 265 \text { infants had positive } \\
\text { SARS-CoV-2 RNA and six of them had } \\
\text { Covid- } 19 \text { positive within first } 12 \text { hours } \\
\text { after birth. }\end{array}$ \\
\hline $\begin{array}{l}\text { (Marin } \\
\text { Gabriel, et } \\
\text { al.,2020) }\end{array}$ & $\begin{array}{l}\text { Descrip } \\
\text { tive } \\
\text { study }\end{array}$ & $\begin{array}{l}\text { Descriptive study/ } \\
242 \text { pregnant women } \\
\text { diagnosed with } \\
\text { Covid-19 }\end{array}$ & $\begin{array}{l}\text { Absence of infant mortality and no vertical } \\
\text { or horizontal transmission was detected. } \\
115(46.3 \%) \text { newborns were in neonatal } \\
\text { unit, among them, } 87(75.6 \%) \text { had exclusive } \\
\text { breast feeding at discharge \& } 40.4 \% \text { of } \\
\text { newborns had feeding at } 1 \text { month. }\end{array}$ \\
\hline $\begin{array}{l}\text { (Zaigham \& } \\
\text { Anderson, } \\
2020),\end{array}$ & Sweden & $\begin{array}{l}\text { Systematic reviewed } \\
108 \text { pregnant with } \\
\text { Covid-19 }\end{array}$ & One neonatal death and fetal death. \\
\hline $\begin{array}{l}\text { (Hassan, } \\
\text { Muzamil \& } \\
\text { Banday } \\
2020)\end{array}$ & India & $\begin{array}{l}\text { Observational study/ } \\
38 \text { pregnant women } \\
\text { with Covid-19 }\end{array}$ & $\begin{array}{l}26.3 \% \text { of newborns had meconium (stool of } \\
\text { fetus) stained, } 29 \% \text { had fetal distress, } 29 \% \\
\text { had ICU admissions, } 13.5 \% \text { had deaths, } \\
(2.7 \%) \text { had intra uterine death, } 2.63 \% \text { had } \\
\text { vertical transmission, } 2.6 \% \text { had lab } \\
\text { characteristics anemia, } 21 \% \text { had } \\
\text { thrombocytopenia, } 21 \% \text { had SARS CoV-2 } \\
\text { positive, } 100 \% \text { was presented with heart } \\
\text { failure and shock at } 17 \text { th day of life. }\end{array}$ \\
\hline $\begin{array}{l}\text { (KC, et al., } \\
2020),\end{array}$ & (Nepal) & $\begin{array}{l}\text { Perspective } \\
\text { observational study } \\
21760 \text { pregnant } \\
\text { mothers without } \\
\text { Covid-19 }\end{array}$ & $\begin{array}{l}\text { Institutional stillbirth were increased } \\
13 / 1000 \text { live births to } 21 / 1000 \text { live birth } \\
\text { and neonatal mortality were increased } \\
13 / 1000 \text { to } 40 / 1000 \text { live births. }\end{array}$ \\
\hline
\end{tabular}

*Total 11article's populations were 2093 pregnant women with Covid-19 but Nepal study population was not included.

\section{Discussion}

The finding of the Meta analysis reveals that $74.16 \%$ of pregnant women with Covid-19 had given birth cesarean section. Less than one third (21.81\%) of pregnant 
mother with Covid-19 had vaginal delivery. The common symptoms of pregnant mother with Covid-19 were fever, cough, dyspnea, and pneumonia severe respiratory distress syndrome, premature delivery, fetal distress, preterm birth. Similar systematic review conducted by Khan, Khan, Mustagir, Rana, Hugue \& Raheman, 2020 result revealed that Covid-19 infected pregnant mother manifested common symptoms as fever $(65 \%)$, cough (38\%), fatigue (15\%) and breathing difficulties (14\%) where as neonatal outcomes as occurrence of preterm birth (29\%) and low birth weight were $16.4 \%$. Present analysis studies show that $26.3 \%$ of newborns had meconium (stool of fetus) stained, 29\% had fetal distress, 29\% had ICU admissions, $13.5 \%$ were died, $(2.7 \%)$ had intra uterine death and $2.63 \%$ had vertical transmission where as similar meta analysis conducted by (Di Mascio, et al., (2020) shows that preterm birth was occurring in $41.1 \%$ of cases and perinatal death was $7.0 \%$. None of the 41 newborns assess showed clinical signs of vertical transmission.

\section{Conclusion}

This Meta analysis concluded that most of the pregnant women with Covid-19, the mode of delivery was cesarean due to maternal Covid related complication and fetal compromise. Severe respiratory distress leaded to ICU admission and ventilator support. The common symptoms of neonates were respiratory distress pneumonia, bacterial pneumonia, low birth, rash asphyxia, DIC, perinatal death and Covid-19 positive.

\section{References}

Albow,J.(2020). New study looks at pregnancy during the Covid-19 pandemic. Oregon:University Oregon. From https://www.aau.edu/researchscholarship/featured-research-topics/new-study-looks-pregnancy-during- covid19-pandemic

Antoun, L., El Taweel, N., Ahmed, I., Patni, S., \& Honest, H. (2020). Maternal COVID-19 infection, clinical characteristics, pregnancy, and neonatal outcome: A prospective cohort study. European Journal of Obstetrics \& Gynecology and Reproductive Biology, 252, 559-562. https://doi.org/10.1016/j.ejogrb.2020.07.008

Blitz, M. J., Rochelson, B., Meirowitz, N., Prasannan, L., Rafael, T. J., Chakravarthy, S., ... \& Chervenak, F. A. (2020). Maternal Mortality Among Women with COVID-19 Admitted to the Intensive Care Unit. Am J Obstet Gynecol.

Chen, H., Guo, J., Wang, C., Luo, F., Yu, X., Zhang, W., Li, J., Zhao, D., Xu, D., Gong, Q., Liao, J., Yang, H., Hou, W., \& Zhang, Y. (2020). Clinical characteristics and intrauterine vertical transmission potential of COVID-19 
infection in nine pregnant women: a retrospective review of medical records. Lancet (London, England), 395(10226), 809-815.https:// doi.org/ 10. 1016/S0140-6736(20) 303603.

Di Mascio, D., Khalil, A., Saccone, G., Rizzo, G., Buca, D., Liberati, M., \& D'Antonio, F. (2020). Outcome of Coronavirus spectrum infections (SARS, MERS, COVID 1-19) during pregnancy: a systematic review and metaanalysis. American journal of obstetrics \& gynecology MFM, 100107.

Favre, G., Pomar, L., Musso, D., \& Baud, D. (2020). 2019-nCoV epidemic: what about pregnancies? Lancet (London, England), 395(10224), e40. https://doi.org/ 10.1016/ S0140-6736(20)30311-1

Hassan, N., Muzamil, M., \& Banday, D., (2020). COVID-19 infection during pregnancy - maternal and perinatal outcomes: a tertiary care centre study. International Journal of Reproduction, Contraception, Obstetrics and Gynecology.9 (9):2020: Https: // dx. doi.org/ 10. 18203/2320-1770.ijrcog 20203853.

K. C., A, Gurung, R., Kinney, M. V., Sunny, A. K., Moinuddin, M., Basnet, O., ... \&Lawn, J. E. (2020). Effect of the COVID-19 pandemic response on intrapartum care, stillbirth, and neonatal mortality outcomes in Nepal: a prospective observational study. The Lancet Global Health, 8(10), e1273-e1281.

Khan, M. M. A., Khan, M. N., Mustagir, M. G., Rana, J., Haque, M. R., \&Rahman, M. M. (2020). COVID-19 infection during pregnancy: a systematic review to summarize possible symptoms, treatments, and pregnancy outcomes. MedRxiv.https://doi.org/10.1101/2020.03.31.20049304

Knight, M., Bunch, K., Vousden, N., Morris, E., Simpson, N., Gale, C., \& Kurinczuk, J. J. (2020). Characteristics and outcomes of pregnant women admitted to hospital with confirmed SARS-CoV-2 infection in UK: national population based cohort study. bmj, 369. 127 (12):1548-1556.https://vimeo.com/ rcog/authorinsights 16403.

Li, N., Han, L., Peng, M., Lv, Y., Ouyang, Y., Liu, K., \& Yang, L. (2020). Maternal and neonatal outcomes of pregnant women with COVID-19 pneumonia: a casecontrol study. Clinical infectious diseases. https://doi.org/10.1093/cid/ciaa352.

Liao, J., He, X., Gong, Q., Yang, L., Zhou, C., \& Li, J. (2020). Analysis of vaginal delivery outcomes among pregnant women in Wuhan, China during the COVID-19 pandemic. International journal of gynaecology and obstetrics: the official organ of the International Federation of Gynaecology and Obstetrics, 150(1), 53-57. https://doi.org/10.1002/ijgo.13188.

Liu, H., Liu, F., Li, J., Zhang, T., Wang, D., \& Lan, W. (2020). Clinical and CT imaging features of the COVID-19 pneumonia: Focus on pregnant women and 
children. The Journal of infection, 80(5), e7-e13. https://doi.org/10.1016/j. jinf. 2020.03.007.

Marín Gabriel, M. A., Reyne Vergeli, M., Caserío Carbonero, S., Sole, L., Carrizosa Molina, T., RiveroCalle, I., Cuadrado Pérez, I., ÁlvarezFernández, B., Forti Buratti, A., Fernández-CañadasMorillo, A., \& Neo-COVID-19 Research Group (2020). Maternal, Perinatal and Neonatal Outcomes with COVID-19: A Multicenter Study of 242 Pregnancies and Their 248 Infant Newborns During Their First Month of Life. The Pediatric infectious disease journal, $\quad$ 10.1097/INF.0000000000002902. Advance online publication. https://doi.org/10.1097/INF.0000000000002902.

Moher, D., Shamseer, L., Clarke, M., Ghersi, D., Liberati, A., Petticrew, M., Shekelle, P., Stewart, L. A., \& PRISMA-P Group (2015). Preferred reporting items for systematic review and meta-analysis protocols (PRISMA-P) 2015 statement. Systematic reviews, 4(1), 1. https://doi.org/10.1186/2046-4053-4-1

Molnar, C., \& Gair, J. (2015). Concepts of Biology: 1st Canadian Edition. Ontario: BC Campus. https://openlibrary-repo.ecampusontario.ca/jspui/handle/ 123456789/ 345.

Rasmussen, S. A., Smulian, J. C., Lednicky, J. A., Wen, T. S., \& Jamieson, D. J. (2020). Coronavirus Disease 2019 (COVID-19) and Pregnancy: What obstetricians need to know? American journal of obstetrics and gynecology, 222 (5):415-426 https: //doi. org/ 10. 1016/j.ajog.2020.02.017.

WHO (2020). New research helps to increase understanding of the impact of COVID19 for pregnant women and their babies. From https:// www.who. int/ news/item/01-09-2020-new-research-helps-to-increase-understanding-of- theimpact-of-covid-19-for-pregnant-women-and-their-babies.

Yu, N., Li, W., Kang, Q., Xiong, Z., Wang, S., Lin, X., \& Chen, S. (2020). Clinical features and obstetric and neonatal outcomes of pregnant patients with COVID-19 in Wuhan, China: a retrospective, single-centre, descriptive study. The Lancet Infectious Diseases. https://doi.org/ 10.1016/S1473-3099 (20)30176-6

Zaigham, M., \& Andersson, O. (2020). Maternal and perinatal outcomes with COVID19: A systematic review of 108 pregnancies. Acta obstetricia et gynecologica Scandinavica, 99(7), 823-829. https://doi.org/10.1111/aogs.13867.

Zimmermann, P., \& Curtis, N. (2020). COVID-19 in children, pregnancy and neonates: a review of epidemiologic and clinical features. The Pediatric infectious disease journal, 39(6), 469-477. 